\title{
Which corticosteroid is a better option for antenatal fetal lung maturation?
}

(c) The Author(s), under exclusive licence to the International Pediatric Research Foundation, Inc 2022

Pediatric Research (2022) 92:915; https://doi.org/10.1038/s41390022-01948-6

We have read the study by Jobe et al., ${ }^{1}$ and wanted to congratulate the authors for this prosperous article and make some minor contributions.

Administration of antenatal corticosteroids (ACS) is well-adopted practice for preterm pregnancies $<34$ weeks' gestation. Either betamethasone or dexamethasone is the recommended ACSs for clinical practice because they do not metabolize by the placental enzymes. Various studies have been performed comparing these two ACSs and reasons for superiority of each drug have been reported, but no study has been done on which one to choose based on the maternal and fetal conditions.

Betamethasone is available in two forms: sodium-phosphate (Beta-P) and acetate (Beta-Ac). Clinical drug is a combination of equal parts of Beta-P ( $3 \mathrm{mg})$ and Beta-Ac $(3 \mathrm{mg})$. Only the sodiumphosphate form can be used intravenously (IV), but both forms can be injected intramuscularly (IM). Beta-P is rapidly dephosphorylated resulting in early high plasma concentrations and short half-life in mother and fetus but Beta-Ac is slowly deacetylated resulting in low peak plasma levels and long half-life. Dexamethasone has a more rapid onset and shorter duration of action than betamethasone. The effect of ACSs on lung cell maturation occurs about 4-6h after drug injection. ${ }^{2}$ Dexamethasone is widely available and low cost, compared with betamethasone.

We suggest that the following should be considered carefully when choosing the type of ACS.

\section{MATERNAL ASPECTS}

1. In mothers that delivery is very imminent (within hours), ACSs with a shorter half-life such as dexamethasone and Beta-P should be given that does not impose long-term effects on mother. Also to achieve an earlier effect of the drug in these cases, it is better to use IV forms that reach their peak plasma level earlier in the fetus.

2. In diabetic mothers, we prefer to use ACS forms with a shorter half-life such as dexamethasone due to their effect on increasing BS.

3. In mothers with rupture of membrane (ROM), it is important to choose the best ACSs due to the increased risk of chorioamnionitis. It seems that ACS with a lower half-life is better to use.

4. In patients with COVID-19 infection, due to the fact that studies have been confirmed the effect of dexamethasone on COVID19 , it is the preferred option. Beta-Ac may not be a suitable option due to its long-lasting effect and the risk of possible superimposed bacterial infections in these patients.
5. In patients with cardiovascular diseases, due to the effects of CSs on the cardiovascular system even at low doses, it is important to pay attention to these effects and do more studies in this area to choose the best option.

\section{FETAL ASPECTS}

1. In fetuses with restricted growth, especially in those with abnormal umbilical Doppler study, we prefer to use ACSs with less complication of stillbirth. Based on Ciapponi ${ }^{3}$ study results, dexamethasone may be a better option, however, more studies are needed.

2. There have been many studies on the effects of ACSs on RDS, IVH, neurodevelopmental disability, and no significant difference has been found between dexamethasone and betamethasone.

Sedigheh Hantoushzadeh ${ }^{1}$, Maasoumeh Saleh ${ }^{{ }^{凶}}$ and Sepehr Aghajanian ${ }^{3}$

${ }^{1}$ Maternal-Fetal Medicine, Department of Obstetrics and Gynecology, Maternal-Fetal Neonatal Research Center, Tehran University of Medical Sciences, Valiasr Hospital, Tehran, Iran. ${ }^{2}$ Maternal-Fetal Medicine, Department of Obstetrics and Gynecology, Tehran University of Medical Sciences, Shariati Hospital, Tehran, Iran. ${ }^{3}$ Department of Community Medicine, School of Medicine, Alborz University of Medical Sciences, Karaj, Iran.

凶email: salehmaasoumeh@yahoo.com

\section{REFERENCES}

1. Jobe, A. H. et al. Antenatal corticosteroids: a reappraisal of the drug formulation and dose. Pediatr. Res. 89, 318-325 (2021).

2. Schmidt, A. F. et al. Dosing and formulation of antenatal corticosteroids for fetal lung maturation and gene expression in rhesus macaques. Sci. Rep. 9, 9039 (2019).

3. Ciapponi, A. et al. Dexamethasone versus betamethasone for preterm birth: a systematic review and network meta-analysis. Am. J. Obstet. Gynecol. MFM 3, 100312 (2021).

\section{COMPETING INTERESTS}

The authors declare no competing interests.

\section{ADDITIONAL INFORMATION}

Correspondence and requests for materials should be addressed to Maasoumeh Saleh.

Reprints and permission information is available at http://www.nature.com/reprints

Publisher's note Springer Nature remains neutral with regard to jurisdictional claims in published maps and institutional affiliations. 